\title{
Enseñanza en ingeniería de manera colaborativa a partir de un diseño tecnopedagógico, usando SMILE
}

\author{
Andrés D. Solano y Marlin A. Aarón \\ Fac. De Ingeniería, Universidad de La Guajira, Km 5 vía Maicao, Riohacha- La Guajira-Colombia \\ (correo-e: andresolano@uniguajira.edu.co, maaron@uniguajira.edu.co)
}

Recibido Dic. 30, 2019; Aceptado Feb. 29, 2020; Versión final Mar.23, 2020, Publicado Ago. 2020

\begin{abstract}
Resumen
Se presenta el resultado de un proceso investigativo que concentra su accionar en cómo realizar una enseñanza que posibilite la comprensión de contenidos. Esto es soportado en una estrategia de indagación para atender las necesidades educativas encontradas en el contexto, con el uso de la plataforma SMILE, que permite construir preguntas y respuestas de forma individual y grupales, logrando así, la colaboración entre estudiantes. La metodología es de corte cuali-cuantitativa. Desde la Investigación Acción se soportó la construcción de las actividades usando la técnica del análisis de contenido, la observación directa y lo cuantitativo, apoyado en técnicas estadísticas. El diseño de la plataforma SMILE posibilitó la evaluación crítica de las construcciones individuales y colectivas, y que los estudiantes se reconocieran como agentes activos y participativos. El diseño tecnopedagógico aportó a las intenciones de los docentes de potenciar el autoaprendizaje y la autogestión de conocimiento en los estudiantes.
\end{abstract}

Palabras clave: diseño tecnopedagógico; plataforma SMILE; aprendizaje colaborativo; ruta pedagógica

\section{Engineering education based on a collaborative techno- pedagogical design, using SMILE}

\begin{abstract}
This study presents the results of an investigative process that concentrates its actions on how to perform a teaching that enhances the understanding of the content presented. This is supported by an inquiry strategy to meet the educational needs found in the context. This study uses the SMILE platform. SMILE's design and functionality allows building questions and answers individually and in groups, which enhances collaboration between students. The methodology is qualitative and quantitative. The Action Research supported the construction of the activities by the technique of content analysis, observation, and quantitative evaluation that was supported by statistical techniques. The design of the SMILE platform enabled the critical evaluation of individual and collective constructions. Students were recognized as active and participatory agents. In conclusion, the techno-pedagogical design contributed to the teachers' intentions of promoting self-learning and self-management knowledge in students.
\end{abstract}




\section{INTRODUCCIÓN}

Las nuevas tecnologías de la información y comunicación en una sociedad caracterizada por un desarrollo tecnológico que avanza a grandes pasos y que reporta importantes beneficios a quienes las utilizan, ha impactado la forma como hoy se enseña y se aprende en el siglo XXI. Esos desarrollos tecnológicos han permeado y retado a los docentes que enseñan en las aulas universitarias, especialmente en ingeniería, debido a que ha demandado que asuman un rol caracterizado por generar y orquestar espacios que respondan a los desafíos actuales en los que se busca lograr la integración de teorías de aprendizajes y las TIC para responder a las intenciones pedagógicas a desarrollar en cualquier espacio formativo y la generación de recursos educativos completos, efectivos y eficaces (Agudelo, 2009) que fortalezcan el aprendizaje. Este nuevo papel del docente ha llevado a recurrir a la combinación de la tecnología y la pedagogía, lo que ha venido fortaleciéndose en los últimos años y se convierte en un aliado para aquellos que buscan mejorar sus estrategias de enseñanza y que surge precisamente por la necesidad de diseñar, planificar y optimizar la manera en la que se dan los procesos de enseñanza y aprendizaje.

La integración racional de la pedagogía y la tecnología puede generar una mezcla que sirve para concebir un diseño tecnopedagógico (Yeh et al., 2017). Este diseño tecnopedagógico DT, (o Instruccional) puede ser entendido como un proceso sistemático para planificar la enseñanza (Berger y Kam, 1996). Para Richey et al. (2001) el DT hace referencia a la "Planificación instruccional sistemática que incluye la valoración de necesidades, el desarrollo, la evaluación, la implementación y el mantenimiento de materiales y programas". Broderick (2001) plantea que "el DT es el arte y la ciencia que permite crear un entorno educativo y sus materiales que lleven al estudiante del estado de no poder realizar ciertas tareas al estado de ser capaz de realizarlas". Por su parte, Coll et al. (2007) plantean que las propuestas de diseño tecnopedagógico "integran tanto los aspectos tecnológicos como los pedagógicos o instruccionales y que adoptan la forma de un diseño tecnopedagógico o tecnoinstruccional". Para los autores este diseño tecnopedagógico fue asumido como un escenario armoniosamente combinado, en la que el componente pedagógico pensado desde los objetivos de enseñanza, se medió usando las tecnologías que posibilitaban la puesta en marcha del mismo. Esto es, el norte lo daba el diseño pedagógico y la tecnología usada como mediación; y ambos, puestos prueba diseño pedagógico y tecnología, se reconstruían a partir de la aplicación conjunta.

Se realiza en una ruta pedagógica puesta en marcha en esta investigación, que se realizó con 44 estudiantes de primer semestre del programa de Ingeniería de Sistemas de la Universidad de La Guajira, soportado lo pedagógico en la teoría constructivista), desde un escenario de indagación donde el estudiante requiere la acción de un agente mediador para acceder a la zona de desarrollo próximo; el docente es responsable de ir tendiendo un andamiaje (Aarón, 2019; Raes et al., 2012; Hederich, 2010), que le proporcione seguridad al estudiante y permita que se apropie del conocimiento y lo transfiera a su propio entorno. Se usó como estrategia didáctica el aprendizaje colaborativo que promueve el aprendizaje centrado en el alumno basando el trabajo en pequeños grupos (Johnson et al., 2000).

La asignatura Introducción a la Ingeniería de Sistemas semestralmente recibe un grupo de estudiantes pertenecientes a los estratos uno y dos, egresados de Instituciones Educativas públicas del departamento de La Guajira que ven a la Universidad de La Guajira como una opción por su bajo costo en comparación con las demás universidades del país. De los estudiantes que cursan la asignatura un pequeño grupo representado en un $8 \%$ son estudiantes provenientes y pertenecientes a comunidades indígenas wayuu, según datos suministrados por los estudiantes en la presentación inicial del curso. Un análisis por observación directa a los estudiantes de los últimos semestres que han cursado la asignatura, ha permitido evidenciar que sus comportamientos tienen características muy similares tales como incumplimiento en la realización de las actividades o tareas propuestas por el docente y a pesar de las reiteradas explicaciones de los temas de clases, en los exámenes escritos presentan falencias. Se les dificulta hacer relaciones de los temas abordados en la asignatura que evidencien sus aprendizajes.

El diseño tecnopedagógico que se presenta, se apoya en lo tecnológico en una mediación para montar el andamiaje, (Badia y García, 2006), la plataforma Stanford Mobile Inquiry-based Learning Environment SMILE, (Kim, 2009; Kim, 2016; Acosta et al., 2014; Aarón, 2019), (Figura 1) que es una plataforma tecnológica diseñada como entorno de aprendizaje basado en la indagación (Suárez, et al, 2018) (Lee, 2012). Esta herramienta tecnológica soporta la ruta pedagógica diseñada para favorecer la acción pedagógica. La plataforma SMILE, de la Universidad de Stanford, entorno virtual al que se accede desde internet y posibilita la construcción de preguntas por parte de todos los estudiantes (Aarón, 2019), son vistas por todos los compañeros de curso y pueden ser respondidas por todos los participantes. Los estudiantes construyen las preguntas y las digitan en la plataforma y también pueden disponer las opciones de respuestas, lo que demanda conocimiento temático, coherencia en la escritura, sentido, comprensión y habilidades tecnológicas. 
Reyes-Cárdenas y Padilla (2012) citando a Novak (1964) señalan que "la indagación es una serie de comportamientos involucrados en los seres humanos para encontrar explicaciones razonables de un fenómeno acerca del cual se quiere saber algo". Asimismo, Reyes-Cárdenas y Padilla (2012) citando a Rutherford (1964) señalan que la indagación "se alcanza cuando el contenido y los conceptos son comprendidos en el contexto de cómo fueron descubiertos y que permitan puedan ocurrir futuras indagaciones" Por su parte Bybee (2004) explica que la enseñanza y el aprendizaje basados en la indagación deben integrar tres componentes: 1) habilidades de indagación (lo que deben hacer los estudiantes); 2) el conocimiento acerca de la indagación (lo que se debe comprender de la naturaleza de la indagación), y 3) una aproximación pedagógica para la enseñanza de los contenidos científicos (lo que deben hacer los docentes). El reto es combinar estos aspectos pedagógicos, la estrategia de indagación y lo tecnológico, la Plataforma SMILE, para posibilitar un DT que dé cuenta del aprendizaje de los contenidos de la asignatura, competencias y habilidades de orden superior.

El aprendizaje basado en indagación (Anderson, 2007; Schwartz 2004; García y Furman, 2014) de acuerdo con Uno (1990) se define como "un método pedagógico que combina actividades de manos en la masa con discusiones centradas en los estudiantes y el descubrimiento de conceptos". Oliveira (2009) afirma que "la enseñanza basada en la indagación es comúnmente definida como un modo instruccional en el que el profesor de ciencia renuncia, al menos parcialmente, a su papel de experto en ciencia al ceder derechos instruccionales como proveer respuestas correctas, decir a los estudiantes qué hacer y evaluar las ideas de los estudiantes". Estos planteamientos dan fuerza al proceso constructivista de indagación desarrollado para este diseño tecnopedagógico, debido a que el proceso formativo pasa a estar centrado en el estudiante comprometiéndolo activamente en su proceso de aprendizaje (Minner et al, 2010) y pone al docente como el facilitador o el guía dentro del proceso de construcción de aprendizajes significativos y diseñador de estrategias para el desarrollo de habilidades de pensamiento (Lim, et al., 2019).

El desarrollo de habilidades de pensamiento se puede lograr por medio de construir preguntas. Para Freire (1986) la pregunta es el eje medular; es el activador del pensamiento y del discurrir sobre los diferentes asuntos que se plantea el grupo como tarea. Para Freire y Faundez (2013) "Las preguntas ayudan a iniciar procesos interactivos de aprendizaje y solución de problemas, lo mismo que mantenerlos hasta cuando se logran los objetivos y se planteen nuevos problemas y nuevas situaciones de aprendizaje en este continuo trasegar que es la vida." La pregunta es, además, un elemento pedagógico que estimula y da solidez al proceso de autoaprendizaje (Furman, 2016; Seol et al., 2011; González et al., 2011; Tapia et al., 2004; Aarón, 2016; Mariño et al., 2004).

El antecedente de esta de investigación que se presenta, se desarrolló en el segundo periodo académico de 2015 donde se pone en marcha en la asignatura Introducción a la Ingeniería de Sistemas, una estrategia de indagación (Aarón, 2019) que surge con un grupo de estudiantes repitentes, en la que se aplica pedagogía activa para eliminar la pasividad del alumno y la memorización de contenidos. Esta estrategia consiste en construir preguntas temáticas que permitan demostrar la comprensión de los contenidos de la asignatura, reconocer el autoaprendizaje durante la construcción de preguntas esenciales derivadas de los contenidos temáticos y lograr la generación de conceptos (Aarón, 2016). Para la puesta en marcha de la estrategia se utilizó como mediación tecnológica, la plataforma SMILE(Stanford Mobile Inquiry-based Learning Environment), de la Universidad de Stanford, que por su diseño y funcionalidad permite al docente crear grupos de trabajos a los que acceden los estudiantes y por medio de recursos llamados actividades, se pueden construir preguntas y respuestas de forma individual y grupales, logrando así la interacción entre ellos respondiendo las preguntas elaboradas por todos, gestándose así un ambiente de indagación en el aula de clases. Como resultado de esta intervención se pudo favorecer un acercamiento significativo en el desarrollo de pensamiento crítico y habilidad analítica, aprobando así la asignatura el $100 \%$ de los estudiantes.

Entre los escenarios en los que ha mediado la investigación en ingeniería para generar modelos de enseñanza situados acompañados por tecnologías, se referencia a Mesa et al. (2018) con su proyecto de actualización de métodos de enseñanza-aprendizaje en asignaturas de Dirección de Proyectos de Ingeniería; a Pineda (2014) con su artículo de modelo tecno-pedagógico basado en ludificación y programación competitiva para el diseño de cursos de programación. Ingeniería de Sistemas e Informática y (Dias, et al., 2014).En ellos, se observa que la incorporación de las nuevas tecnologías facilitó la construcción del diseños tecnopedagogicos para favorecer el trabajo colaborativo de los alumnos y las tareas de seguimiento y evaluación de los docentes.

El proyecto de investigación sobre el que está soportado este artículo, concentra su accionar en cómo realizar una enseñanza que posibilite la comprensión de contenidos, apoyado en una estrategia de indagación, usando la plataforma SMILE. La cuestión central aquí, fue identificar como desde el diseño tecnopedagógico posibilitó atender las necesidades educativas encontradas, usando la Plataforma SMILE y potenciar una acción pedagógica en contexto. 


\section{METODOLOGIA}

La metodología usada es de corte cuali-cuantitativa. Desde la investigación acción se soportó la construcción de actividades de clases, usando técnicas de análisis de contenido y observación directa. Para lo cuantitativo, se apoyó en técnicas estadísticas. Lo tecnológico, desde el ciclo de vida del sistema. De manera específica el diseño tecnopedagógico se sustenta desde la investigación acción participación, (Balcazar, 2003; Fals Borda,1970), orientada hacia el cambio educativo y lo cuantitativo, construcción y análisis de formatos de valoración para cada sesión de clases que se aplicaban a los estudiantes, como un ejercicio permanente que permitía ir construyendo un avance de los participantes en cuanto a la comprensión de los temas y la elaboración de preguntas, se soportó en las técnicas estadísticas.

Para construir la secuencia didáctica, se utilizó la investigación acción y eso se logró por medio de un formato de valoración de cada sesión de clases, que indagó sobre los avances y la valoración que hacían los estudiantes de su propio progreso en el desarrollo de la actividad de cada sesión, de sus habilidades tecnológicas en la plataforma y sobre la construcción de las preguntas. El grupo de preguntas consignadas en el formato eran: ¿Te parece fácil acceder y usar la plataforma SMILE? ¿Cómo te parecieron las preguntas? ¿Fueron comprendidas por ti? ¿Las respuestas como te parecieron? ¿Los exámenes usando la Plataforma SMILE, te parecen que ayudan a comprender los temas? Esto permitía observar cómo había sido valorada por los estudiantes la actividad que se había dispuesto; si esto había contribuido para fortalecer sus aprendizajes y la construcción de las preguntas temáticas. Cada acción formativa desarrollada dentro de la ruta pedagógica tuvo un formato en el que las preguntas iban en consonancia con lo desarrollado en la actividad y tenían como centro las apreciaciones de los estudiantes sobre la construcción de preguntas, respuestas, cómo valoraban el aporte de sus compañeros, la consolidación de sus conocimientos y recomendaciones para mejorar el desarrollo de la actividad.

smile A MyGroups Consortium

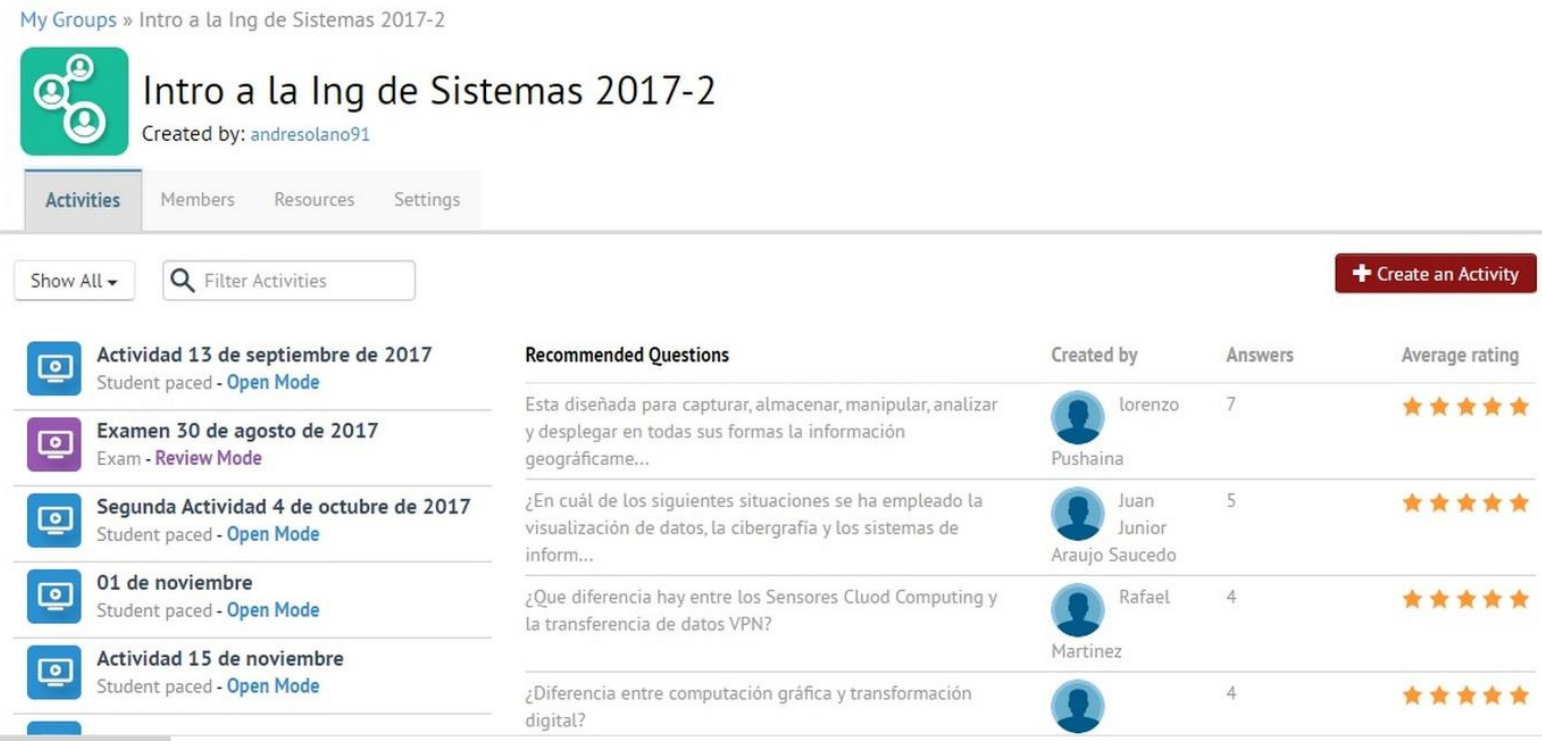

Fig. 1: Entorno grafico del espacio para la asignatura en SMILE

El diseño tecnopedagógico se desarrolló en una secuencia didáctica de siete (7) actividades, que también se apoyaba tecnológicamente en la plataforma virtual institucional Akumaja, Ambiente virtual de aprendizaje de la Universidad de la Guajira soportada en Moodle; desde allí se disponía el acceso a la Plataforma Smile. Las actividades fueron: 1) Preguntas elaboradas por los docentes y dispuestas en la Plataforma SMILE, 2) Respuesta a preguntas elaboradas por los docentes en SMILE; 3) Construcción individual de preguntas en la plataforma SMILE; 4) uso de rubricas para evaluar preguntas construidas por estudiantes en SMILE; 5) Reconstrucción de preguntas entre pares en SMILE; 6) Evaluación de pares a las preguntas construidas en SMILE y 7) Construcción colaborativa de preguntas y respuestas en SMILE. Todas estas fueron soportadas por las prestaciones tecnológicas de la Plataforma SMILE que permite la creación de sesiones para las construcciones individuales y grupales de preguntas y de respuestas, o solo preguntas, o solo respuestas, que acorde a la actividad serían construidas por docentes o por estudiantes. Las decisiones de si solo unas u otras, o ambas al mismo tiempo, eran tomadas por los docentes, a partir del análisis de cada sesión.

El escenario virtual en la plataforma SMILE se inicia al crear un grupo; para este caso tiene el nombre de la asignatura, como se observa en la Figura 1. Este es el entorno tecnológico en el que los estudiantes pueden 
acceder para vivenciar el componente pedagógico que es la estrategia de indagación y elaborar las preguntas y las respuestas acorde a la ruta pedagógica que se diseña. La plataforma SMILE se convierte en la mediación tecnológica que posibilita el entorno pedagógico. De allí la necesidad de valorar sus potencialidades y articulación, lo que se hacía a través del formato de evaluación de clases, que diligenciaban los estudiantes al final de esta. Después de crear el grupo, para configurar la sesión de preguntas individuales el docente desde la plataforma SMILE, seleccionaba el tipo de recurso que se adaptaba a la actividad que ha diseñado. La plataforma contiene varios recursos que se convierten en prestaciones tecnológicas que median la acción pedagógica.

Las actividades realizadas en la plataforma SMILE que se ejecutaban en cada sesión de clases, pretendían potenciar habilidades de pensamiento de orden superior, competencias comunicativas, digitales. Se pretendía que los estudiantes construyeran preguntas de conocimiento, comprensión de contenido, aplicación y análisis (Sadker y Sadker, 1998). Cada una de las preguntas significaba para ellos un esfuerzo intelectual en el sentido que debían asociar el contenido temático con la elaboración de preguntas acorde a cada tipo de ellas, siendo las de conocimiento, preguntas fácticas tales como que es esto o aquello, y las de análisis, preguntas que buscaban que los estudiantes no solo conociesen significados y ocurrencia de eventos, sino que hiciesen inferencias o generalizaciones a partir de ello.

Las actividades del DT usaban recursos de la plataforma SMILE que fueron seleccionándose a partir de sus prestaciones. En la actividad 1 y 2 se utilizó el recurso exam, que fue útil para que los docentes crearan las preguntas para diagnosticar las habilidades que tenían los estudiantes para la confección de las preguntas. Este estilo de recurso examen requiere que se asigne un nombre, una descripción y seleccionar una opción para que quede visible la actividad; estos son los elementos mínimos necesarios para habilitar este recurso. En el modo de examen, solo los organizadores de grupos (docentes) pueden crear preguntas. La calificación y los comentarios están deshabilitados. La sesión permanece cerrada (las preguntas no son visibles) hasta que ingresan al modo de resolución, es decir cuando los estudiantes tienen habilitadas las preguntas para responderlas. Los resultados están ocultos hasta que se muestran.

Antes de realizar la actividad 3 en la Plataforma SMILE, se invitó a los estudiantes a realizar un ejercicio preparatorio a la misma. Se les pidió construir preguntas temáticas, usando un documento en Microsoft Word con la intención de que ellos se iniciaran en la elaboración de preguntas; esta actividad se organiza para trabajarla en parejas. Como producto se esperaba que quedara un banco de preguntas que luego deberían ser subidas a la plataforma. Durante la actividad los estudiantes manifestaban su deseo y ansias por trasladar las preguntas escritas en Word en la Plataforma SMILE. El recurso Open to closed-ended, fue utilizado para la actividad 3. Este es un cuestionario en el que los estudiantes primero crean preguntas abiertas y luego agregan opciones de respuesta, responden y califican las preguntas de los demás. El organizador (el docente) controla cuándo los estudiantes pueden crear preguntas y cuándo pueden responder preguntas. La sesión permanece abierta desde el momento de su creación y los comentarios están habilitados para que los estudiantes hagan la valoración a lo elaborado por sus compañeros teniendo como guía la rúbrica diseñada y dispuesta en clase por los docentes.

En la secuencia didáctica finalmente se utilizó para las actividades 4,5 y 6 el recurso Student paced. Este tipo de recurso es una prueba abierta en la que los estudiantes primero crean sus propias preguntas y luego responden y califican las preguntas de los demás. Los estudiantes pueden crear y responder preguntas al mismo tiempo. La sesión permanece abierta desde el momento de su creación y los comentarios están habilitados para que los estudiantes trabajen colaborativamente y den sus aportes para afinar las preguntas elaboradas por sus compañeros. Estas actividades unidas con el recurso presentado permite generar trabajo colaborativo entre los estudiantes. En estas actividades los estudiantes de manera colaborativa construyeron sus preguntas en la plataforma SMILE, lo que les ayudó a que mejorara sustancialmente la forma de construir preguntas, con una mayor lógica y claridad de los conceptos que se habían abordado en clases.

Durante estas sesiones de clases se usa la plataforma SMILE por parte de los estudiantes. Las técnicas utilizadas para la recolección de información por parte de los docentes durante la sesión, son la observación directa a partir de lo que ocurre en aula y entrevistas que realizan los docentes a los estudiantes en una actividad de reflexión de diálogo abierto. La observación directa, las entrevistas con dialogo abierto y el análisis al formato de valoración que realizan los estudiantes al final de cada la actividad, se convierten en las bases sobre las que se soporta la construcción y reconstrucción de la ruta pedagógica, toda vez que esta es objeto de reflexión al finalizar cada sesión a partir de los hallazgos que resultan de estas técnicas.

\section{RESULTADOS Y DISCUSIÓN}

En cuanto a las tecnologías con que cuentan los estudiantes, su acceso en tiempo libre y su percepción sobre la facilidad para ello, se presentan estos datos que fueron tomados del formato de valoración. Los estudiantes 
indican que las tecnologías con que disponen son: Smartphone (49\% de los estudiantes), Tablet (35\% de los estudiantes) y un $9 \%$ no contaban con tecnología. En cuanto al acceso a la plataforma SMILE, al $100 \%$ de los estudiantes les pareció fácil y todos manifestaron que podían accesarla en su tiempo libre. La secuencia didáctica de las mismas y sus resultados se muestran en la Tabla 1. En la Tabla 2 se puede observar que en las actividades 1 y 2 , los docentes elaboraron 4 preguntas sobre los temas que se habían abordado hasta ese momento con los estudiantes. Entre estos estaban, Ciencia, Ingeniería, Sistemas, Informática y Computación. En esta actividad los docentes buscaban dos cosas, uno, que estas preguntas y opciones de respuesta fueran un referente para los estudiantes sobre cómo podrían construirse preguntas de análisis y dos, identificar claridades conceptuales por parte de los estudiantes de los temas abordados hasta esa fecha para observar logros en los objetivos de aprendizaje, porque a los estudiantes se les pidió resolver las preguntas, al finalizar la presentación temática.

Tabla 1: Secuencia didáctica de las actividades dentro del diseño tecnopedagógico

\begin{tabular}{|c|c|c|c|c|c|c|}
\hline № & $\begin{array}{l}\text { Nombre de la } \\
\text { Actividad }\end{array}$ & $\begin{array}{l}\text { Momento en que } \\
\text { se realizó la } \\
\text { actividad en SMILE }\end{array}$ & $\begin{array}{l}\text { Recurso } \\
\text { usado en la } \\
\text { plataforma }\end{array}$ & $\begin{array}{l}\text { Autor de } \\
\text { las } \\
\text { preguntas }\end{array}$ & $\begin{array}{l}\text { Cantidad de } \\
\text { preguntas en } \\
\text { la plataforma }\end{array}$ & $\begin{array}{l}\text { Cantidad de } \\
\text { respuestas de } \\
\text { los estudiantes }\end{array}$ \\
\hline 1 & $\begin{array}{l}\text { Creación de } \\
\text { preguntas por los } \\
\text { docentes }\end{array}$ & $\begin{array}{l}\text { Clase 1: agosto } \\
2017 .\end{array}$ & Exam & Docentes & 4 & $\mathrm{Na}$ \\
\hline 2 & $\begin{array}{l}\text { Respuesta a } \\
\text { preguntas elaboradas } \\
\text { por los docentes }\end{array}$ & $\begin{array}{l}\text { En clases, antes } \\
\text { del primer parcial }\end{array}$ & Exam & Docentes & 4 & 177 \\
\hline 3 & $\begin{array}{l}\text { Construcción } \\
\text { individual de } \\
\text { preguntas }\end{array}$ & $\begin{array}{l}\text { En clases, antes } \\
\text { del primer parcial }\end{array}$ & $\begin{array}{l}\text { Open to } \\
\text { closed- } \\
\text { ended }\end{array}$ & Estudiantes & 64 & 159 \\
\hline 4 & $\begin{array}{l}\text { Uso de rubricas para } \\
\text { evaluar preguntas } \\
\text { construidas por } \\
\text { estudiantes }\end{array}$ & $\begin{array}{l}\text { En clases, antes } \\
\text { del segundo } \\
\text { parcial }\end{array}$ & $\begin{array}{l}\text { Open to } \\
\text { closed- } \\
\text { ended }\end{array}$ & Estudiantes & 38 & 159 \\
\hline 5 & $\begin{array}{l}\text { Reconstrucción de } \\
\text { preguntas entre pares } \\
\text { en Smile }\end{array}$ & $\begin{array}{l}\text { En clases, antes } \\
\text { del segundo } \\
\text { parcial }\end{array}$ & $\begin{array}{l}\text { Student } \\
\text { paced }\end{array}$ & Estudiantes & 38 & 194 \\
\hline 6 & $\begin{array}{l}\text { Evaluación de pares a } \\
\text { las preguntas } \\
\text { construidas en Smile }\end{array}$ & $\begin{array}{l}\text { En clases, antes } \\
\text { del tercer parcial }\end{array}$ & $\begin{array}{l}\text { Student } \\
\text { paced }\end{array}$ & Estudiantes & 28 & 409 \\
\hline 7 & $\begin{array}{l}\text { Construcción colabo- } \\
\text { rativa de preguntas y } \\
\text { respuestas }\end{array}$ & $\begin{array}{l}\text { En clases, antes } \\
\text { del examen }\end{array}$ & $\begin{array}{l}\text { Student } \\
\text { paced }\end{array}$ & Estudiantes & 80 & 638 \\
\hline
\end{tabular}

El análisis de lo realizado en la actividades 1 y 2 , lo desarrollado sobre construcción de preguntas en el banco de pregunta en Microsoft Word y lo consignado por los estudiantes en el formato de valoraciones, como se muestra en la tabla 2, permitió a los docentes proponer las actividades 3 y 4 en dos sesiones diferentes en la plataforma SMILE que ayudara a los estudiantes en la elaboración de sus preguntas de conocimiento, de comprensión, aplicación, análisis y se buscaba que con la rúbrica cada estudiante pudiera autoevaluarse y valorar el progreso de sus compañeros con los respecto a las preguntas que elaboraban. En la Tabla 1, además se puede ver avance en la cantidad de preguntas y respuestas desarrolladas por los estudiantes en las actividades 5, 6 y 7, estas actividades propiciaron la participación y colaboración de todos como elemento fundamental para realizar la construcción de preguntas con éxito. Los estudiantes fueron activos e interactuaron estudiantes-estudiantes, estudiantes-docente y lo más importante desarrollaron habilidades y destrezas para autodirigirse, seleccionar información necesaria y trabajar en equipo. Ante esto se pudo evidenciar lo significativo que fue trabajar en duplas para la construcción de buenas preguntas, en la que los estudiantes conformarían equipos para trabajar en colaboración y dialogar entre ellos y con el docente, para construir conocimiento y profundizar la comprensión.

Tabla 2: Tipo de preguntas creadas en el transcurso de la secuencia didáctica

\begin{tabular}{|l|r|r|r|r|r|r|r|}
\hline $\begin{array}{l}\text { Tipos de } \\
\text { preguntas }\end{array}$ & $\begin{array}{l}\text { Actividad 1 } \\
\text { (Ag./2017) }\end{array}$ & $\begin{array}{l}\text { Actividad 2 } \\
\text { (Ag./2017) }\end{array}$ & $\begin{array}{l}\text { Actividad 3 } \\
\text { (Sept./2017) }\end{array}$ & $\begin{array}{l}\text { Actividad 4 } \\
\text { (Sept./2017) }\end{array}$ & $\begin{array}{l}\text { Actividad 5 } \\
\text { (Oct/2017) }\end{array}$ & $\begin{array}{l}\text { Actividad 6 } \\
\text { (Oct/2017) }\end{array}$ & $\begin{array}{l}\text { Actividad 7 } \\
\text { (Nov./2017) }\end{array}$ \\
\hline Conocimiento & 0 & 0 & 38 & 8 & 8 & 0 & 5 \\
\hline Comprensión & 0 & 0 & 26 & 28 & 2 & 2 & 11 \\
\hline Aplicación & 0 & 0 & 0 & 0 & 0 & 8 & 20 \\
\hline Análisis & 4 & 4 & 0 & 0 & 45 \\
\hline
\end{tabular}


El análisis del tipo de preguntas que construyen los estudiantes es un indicador del nivel cognitivo y además puede ayudar a evidenciar si hay falencias en el aprendizaje, específicamente en las habilidades de pensamiento crítico, analítico y comunicativas, que deben ser fortalecidas y ayudar a los estudiantes a transitar hasta la consolidación de un aprendizaje profundo. Recuérdese que las actividades 1 y 2 , fueron preguntas construidas por los docentes y respondidas por lo estudiantes. En la Tabla 2, se muestra como en la actividad 3, hay una inclinación inicial por los estudiantes a la construcción de preguntas de conocimiento (ver figura 2), que representaron un $59 \%$ de las preguntas elaboradas en esa sesión y el otro $41 \%$ construyeron preguntas que se acercaban a preguntas de comprensión. La actividad 3 se convierte en el reflejo de las comprensiones iniciales de los estudiantes a los temas abordados hasta la fecha y evidenciaba un mapa de sus habilidades analíticas y críticas, desde la construcción de preguntas.

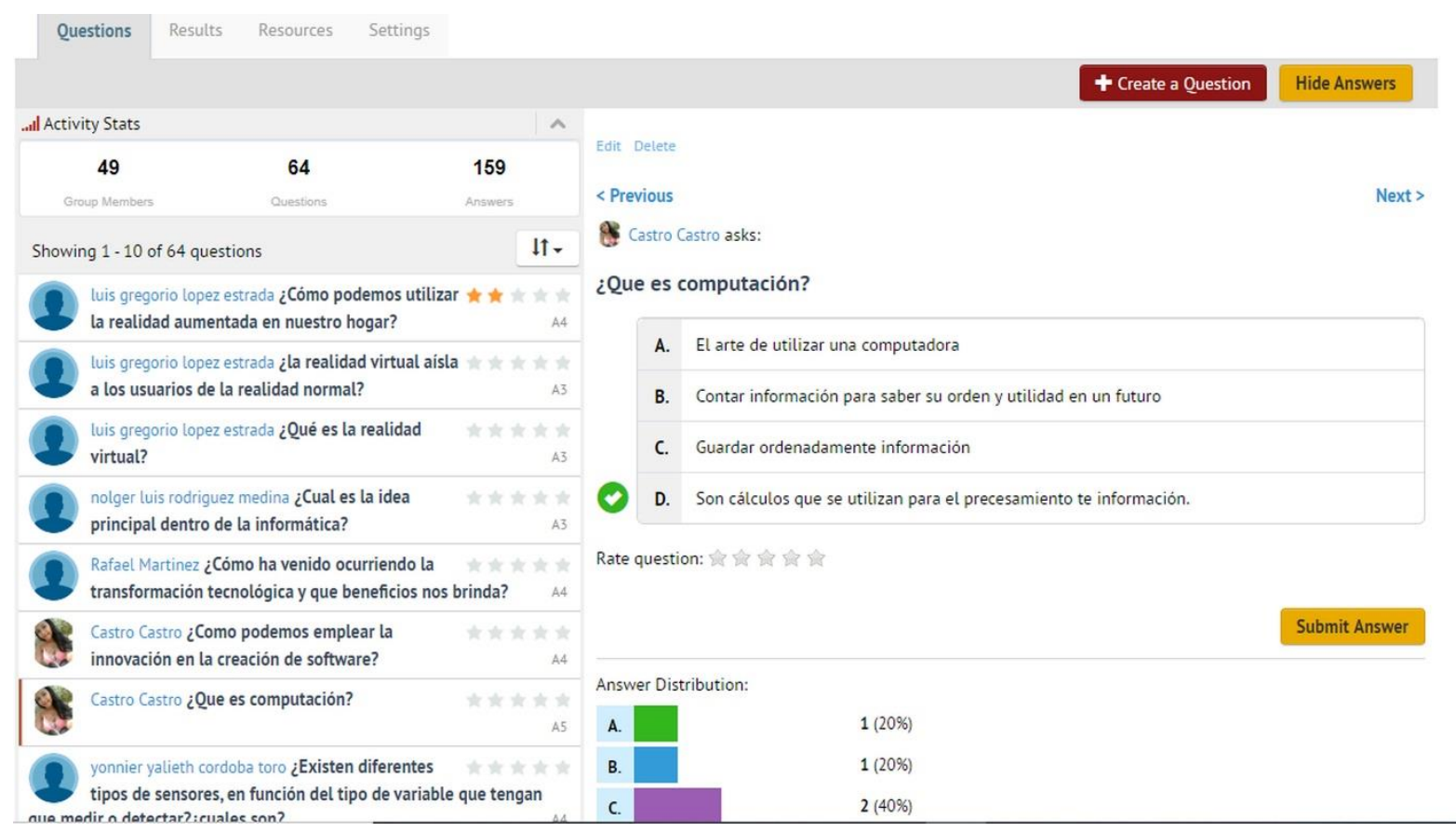

Fig. 2. Actividad colaborativa en la plataforma SMILE. Preguntas de conocimiento tomadas de la Actividad 3.

En la tabla 2, se muestra como los estudiantes van mejorando sustancialmente en la forma de construir preguntas con una mayor lógica y mayor claridad de conceptos abordado en clases. Esto se observa también a partir de la forma como van construyendo las respuestas. Como producto de las actividades los estudiantes construyeron un total de 65 preguntas de diferentes niveles entre las que había de comprensión, aplicación, análisis y de pensamiento crítico. Un ejemplo se muestra en la figura 3.

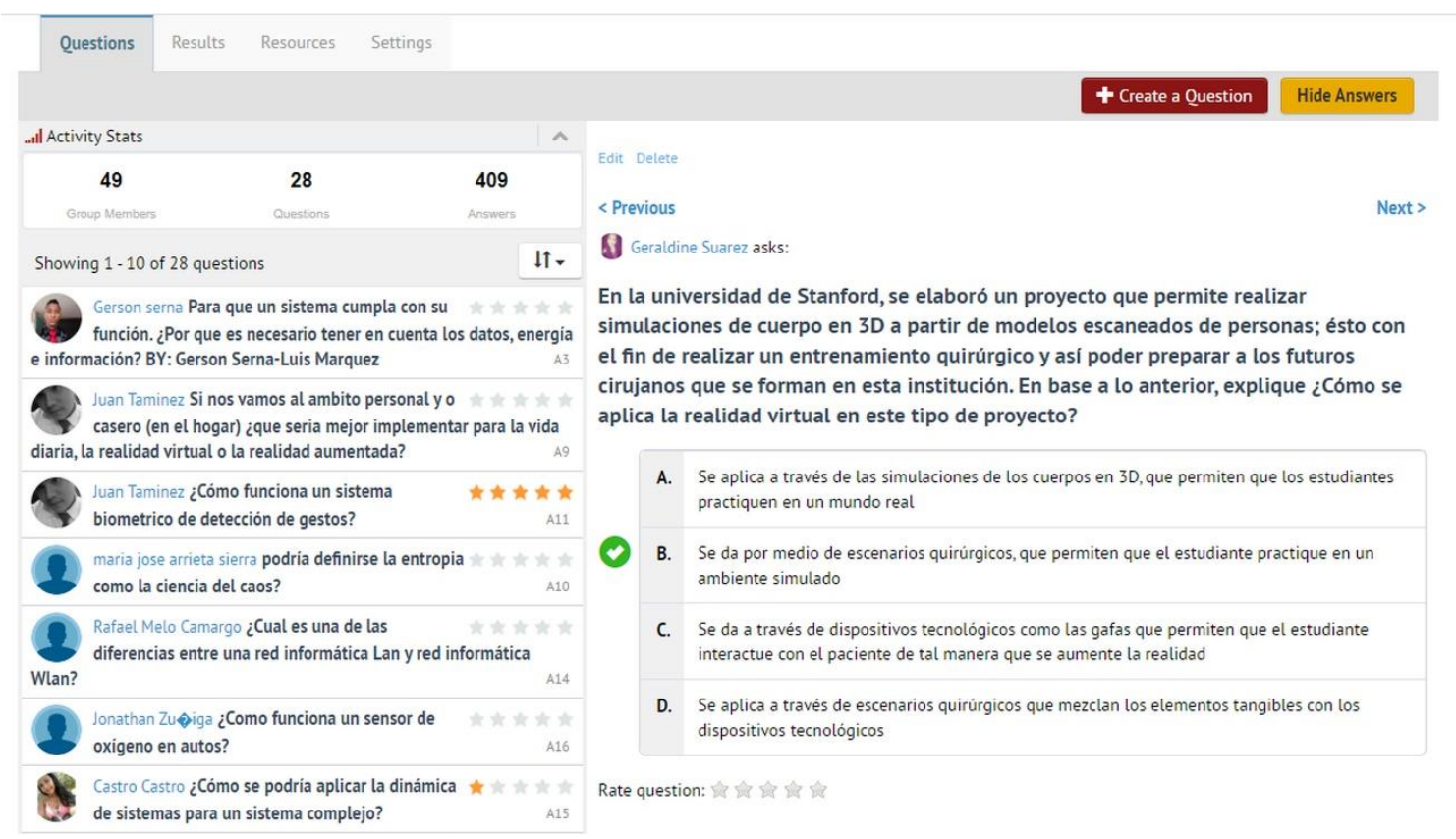

Fig. 3. Pregunta de análisis creada por los estudiantes. Preguntas de análisis tomada de la Actividad 7. 
El análisis de contenidos permitió la construcción de categorías de análisis usando como sustento las respuestas dejadas por los estudiantes en los Formatos de evaluación de las sesiones de clases. Las clases se iniciaban con repaso, presentaciones temáticas por estudiantes y profesores, luego las actividades y al final la evaluación de la sesión. Los estudiantes completaban las actividades en sus casas, si no alcanzaban a completarlas durante la clase, pero siempre se les instaba a realizarlas inmediatamente. Se construyeron 14 categorías de análisis, que se listan a continuación, Fácil construcción de preguntas, Profundidad en el conocimiento, Conocimiento previo, Trabajo Colaborativo, Dificultad en la construcción de Respuestas, Dominio temático, Profundidad en el pensamiento, Creatividad y análisis, Complicada construcción de preguntas, Evaluación crítica a los compañeros, Contenido de las preguntas, Relación preguntas comprensión de temas, Rubricas como apoyo para aprender, Uso y Valor de la Plataforma SMILE En la Tabla 3 se observan algunas de las valoraciones de los estudiantes.

Tabla 3: Algunas valoraciones realizada por los estudiantes a las actividades desarrolladas en la asignatura

\begin{tabular}{|c|c|c|c|c|}
\hline $\begin{array}{l}\text { Nombre de la } \\
\text { Actividad }\end{array}$ & \multicolumn{2}{|l|}{ Valoración de los estudiantes } & \multicolumn{2}{|c|}{ Categorías de análisis } \\
\hline $\begin{array}{l}\text { Creación de } \\
\text { preguntas por los } \\
\text { docentes }\end{array}$ & \multicolumn{2}{|c|}{$\begin{array}{l}\text { "Esta actividad se convierte en un referente de cómo } \\
\text { elaborar preguntas cuidando que ellas tuvieran los } \\
\text { elementos necesarios para no desviar sobre lo que se } \\
\text { estaba preguntando" }\end{array}$} & \multicolumn{2}{|c|}{ Contenido de las preguntas } \\
\hline $\begin{array}{l}\text { Respuesta a } \\
\text { preguntas } \\
\text { elaboradas por } \\
\text { los docentes en } \\
\text { Smile }\end{array}$ & $\begin{array}{l}\text { "Me pareció excelente el uso de } \\
\text { la plataforma SMILE ya que uno } \\
\text { va aprendiendo más con esas } \\
\text { dinámicas de crear preguntas y } \\
\text { ayuda a comprender más los } \\
\text { textos y aprender más" }\end{array}$ & $\begin{array}{l}\text { "Es necesario seguir } \\
\text { realizando actividades } \\
\text { en la plataforma smile" }\end{array}$ & $\begin{array}{l}\text { Relación } \\
\text { preguntas } \\
\text { comprensión } \\
\text { de temas }\end{array}$ & $\begin{array}{l}\text { Uso y Valor } \\
\text { de la } \\
\text { Plataforma } \\
\text { SMILE }\end{array}$ \\
\hline $\begin{array}{l}\text { Construcción } \\
\text { individual de } \\
\text { preguntas en la } \\
\text { plataforma smile }\end{array}$ & \multicolumn{2}{|c|}{$\begin{array}{l}\text { "Fue interesante, porque muchos creíamos que sabíamos } \\
\text { elaborar una pregunta, pero en si no era así, ya que a la } \\
\text { hora de la evaluación hecha por el docente, hubo muchas } \\
\text { dificultades al realizar los diferentes tipos de preguntas". }\end{array}$} & \multicolumn{2}{|c|}{$\begin{array}{l}\text { Complicada construcción de } \\
\text { preguntas }\end{array}$} \\
\hline $\begin{array}{l}\text { Uso de rubricas } \\
\text { para evaluar } \\
\text { preguntas } \\
\text { construidas por } \\
\text { estudiantes en } \\
\text { Smile }\end{array}$ & $\begin{array}{l}\text { "Un buen método para poder } \\
\text { corregir nuestras falencias, en } \\
\text { dicho tema" }\end{array}$ & $\begin{array}{l}\text { "Por medio de las } \\
\text { rubricas me pueden } \\
\text { dar que estaba } \\
\text { equivocado y debía } \\
\text { corregir" }\end{array}$ & $\begin{array}{l}\text { Complicada } \\
\text { construcción } \\
\text { de preguntas }\end{array}$ & $\begin{array}{l}\text { Rubricas como } \\
\text { apoyo para } \\
\text { aprender }\end{array}$ \\
\hline $\begin{array}{l}\text { Reconstrucción } \\
\text { de preguntas } \\
\text { entre pares en } \\
\text { smile }\end{array}$ & \multicolumn{2}{|c|}{$\begin{array}{l}\text { "Me pareció excelente porque con eso recordamos tanto la } \\
\text { clase vista anterior como la forma exacta de construcción } \\
\text { de preguntas llevando en la plataforma smile", }\end{array}$} & \multicolumn{2}{|c|}{ Contenido de las preguntas } \\
\hline $\begin{array}{l}\text { Evaluación de } \\
\text { pares a las } \\
\text { preguntas } \\
\text { construidas en } \\
\text { Smile }\end{array}$ & \multicolumn{2}{|c|}{$\begin{array}{l}\text { "Interesante, interactuar junto con los compañeros, ayuda a } \\
\text { la hora de elaborar las preguntas, ya que, si hay algún } \\
\text { error, él puede ayudarte a corregirlo". }\end{array}$} & \multicolumn{2}{|c|}{ Trabajo Colaborativo } \\
\hline $\begin{array}{l}\text { Construcción } \\
\text { colaborativa de } \\
\text { preguntas y } \\
\text { respuestas }\end{array}$ & $\begin{array}{l}\text { "Nunca había trabajo por medio } \\
\text { de una plataforma y con la } \\
\text { estrategia de Introducción, } \\
\text { aprendí mucho". }\end{array}$ & $\begin{array}{l}\text { "Ha sido importante los } \\
\text { métodos aplicados } \\
\text { como el b-learning y la } \\
\text { plataforma SMILE" }\end{array}$ & \multicolumn{2}{|c|}{$\begin{array}{l}\text { Uso y Valor de la Plataforma } \\
\text { SMILE }\end{array}$} \\
\hline
\end{tabular}

El diseño de los exámenes parciales y final también se elaboraban incluyendo preguntas y respuestas de opción múltiple, la mayoría de análisis, usando incluso preguntas realizadas por los estudiantes. Luego de realizadas las siete (7) actividades en la plataforma SMILE, se registran los resultados de los estudiantes con respecto al examen final. El $79 \%$ de los estudiantes obtuvieron una nota aprobatoria y un 14\% no superó dicho examen. El desempeño académico de los estudiantes en la trayectoria de la asignatura, se muestra en la Tabla 4. Se buscaba identificar en este grupo de estudiantes como a través de la construcción de preguntas y respuestas pudieran demostrar sus aprendizajes adquiridos a lo largo de la asignatura.

Tabla 4: Resultado de los parciales de la asignatura Introducción a la Ingeniería de Sistemas

\begin{tabular}{|l|l|l|l|l|l|l|}
\hline Resultado parciales & \multicolumn{2}{|l|}{ Primer parcial } & \multicolumn{2}{l|}{ Segundo parcial } & \multicolumn{2}{l|}{ Examen final } \\
\hline Aprobados & 11 & $25 \%$ & 28 & $64 \%$ & 35 & $79 \%$ \\
\hline No aprobados & 33 & $75 \%$ & 16 & $36 \%$ & 6 & $14 \%$ \\
\hline Retirados & & & & & 3 & $7 \%$ \\
\hline
\end{tabular}


Finalmente, estas actividades realizadas con la plataforma SMILE valida lo que Coll et al. (2007) expresan sobre la integración de las TIC en el proceso pedagógico "las TIC pueden desplegar su máxima capacidad mediadora como instrumentos psicológicos cuando son utilizadas como "instrumentos cognitivos". Ellas pudieron ayudar a mediar las relaciones entre los participantes (docentes- estudiantes- estudiantes), contenidos de aprendizajes; fueron resultado de una intencionalidad pedagógica, sin ser el centro del proceso sino potenciadoras de lo que los estudiantes debían aprender.

Es importante valorar las intenciones de los investigadores Gonzales y Díaz en su documento, "Integración de la metodología basada en la indagación para mediar la enseñanza de la asignatura Fundamentos de Administración soportada con la herramienta Smile", (González y Díaz, 2015), en la que uno de los objetivos de su investigación fue "superar los procesos de pensamiento memorístico desarrollando actividades que comprometan un alto nivel cognitivo apoyados en la taxonomía de Bloom (analizar, comprender y crear), lo que va en consonancia con los resultados de este diseño tecnopedagógico que buscó convertirse en un instrumento cognitivo para aprender, como apoyo a la pretensión pedagógica. Reconocemos los resultados investigativos de Song et al., (2012), quienes en su proyecto, "Inquiry-based learning environment using mobile devices in math classroom", expresan que SMILE sirvió para involucrar a los estudiantes en clases que promovían habilidades heterogéneas / mixtas, la colaboración en equipo y la competencia en el aprendizaje de las matemáticas.

\section{CONCLUSIONES}

A partir de los resultados obtenidos, se pueden extraer las siguientes conclusiones:

1) El diseño tecnopedagógico que se planeó sirvió para atender la estrategia de indagación y se convirtió en una mediación entre los docentes y los estudiantes porque posibilitó que los estudiantes realizaran las tareas planeadas. El uso de la plataforma SMILE aportó al diseño tecnopedagógico, toda vez que las pretensiones de los docentes de contar con un entorno para la construcción de preguntas se favoreció a partir de las prestaciones con que cuenta la plataforma tecnológica; 2) La ruta pedagógica diseñada e implementada en el curso posibilitó la comprensión de contenidos en los estudiantes permitiéndose con ello atender las necesidades educativas encontradas; 3) Las potencialidades tecnológicas de la plataforma SMILE en conjunto con las actividades diseñadas se convirtieron en un entorno motivante para los estudiantes de la asignatura. Los estudiantes podían tener mayor motivación al pensar preguntas temáticas en un entorno tecnológico que en una hoja Microsoft Word y además retarse a responder las preguntas diseñadas por sus compañeros y observar a través de la plataforma sus resultados; 4) Se considera que la plataforma SMILE dentro del diseño tecnopedagógico aportó a las intenciones de los docentes de potenciar el autoaprendizaje, la autogestión de conocimiento desde lo colaborativo y lo individual; 5) Una buena manera de atender las necesidades particulares de un contexto es crear actividades que sean resultado de la reflexión sobre lo que va ocurriendo y la valoración que los mismos estudiantes hacen de su proceso, lo cual promueve la integración entre actores, contenidos y contexto, posibilitándoles a los estudiantes la aplicación, comprensión de los temas que son objeto de estudio; 6) Si bien un bajo nivel de los estudiantes (16\%), no contaba con tecnología propia para acceder a la Plataforma Smile, todos manifestaron no tener ninguna dificultad para desarrollar las actividades dispuestas para casa, lo que le permitió a los docentes generar las didácticas de integración de la pedagogía y la tecnología, con extensión al tiempo fuera de clases; y 7) Las técnicas usadas para articular la comprensión de los contenidos y la construcción de preguntas permitieron a los estudiantes hacer uso de las zonas de desarrollo próximo para lograr establecer las relaciones que posibilitaron la construcción de conocimiento.

\section{REFERENCIAS}

Aarón, M.A., Uso de la Plataforma Smile para la Elaboración de Conceptos en Estudiantes en Repitencia en un Programa de Ingeniería. http://dx.doi.org/10.4067/S0718-07642019000200265, Información tecnológica, 30(2), 265-274. (2019)

Acosta, O.C., Behar, P. A. y Reategui, E.B., Content recommendation in an inquiry-based learning environment. In Frontiers in Education Conference FIE, 1-6 (2014)

Agudelo, M., Importancia del diseño instruccional en ambientes virtuales de aprendizaje. Nuevas ideas en informática educativa, 5, 118-127. (2009)

Anderson, R.D., Inquiry as an organizing theme for science curricula. En: Abell, S. K., Lederman, N. G. (eds.), Hand book of Research on Science Education, pp. 808-830. New York: Routledge, (2007)

Badia, A., García, C. Incorporación de las TIC en la enseñanza y el aprendizaje basados en la elaboración colaborativa de proyectos. Revista de Universidad y Sociedad del Conocimiento, 3 (2) (2006)

Balcazar. F.E., Investigación acción participativa (iap): Aspectos conceptuales y dificultades de implementación. Fundamentos en humanidades, 4(7-8), 59-77. (2003) 
Berger, C., y Kam, R., Definitions of Instructional Design. Adapted from" Training and Instructional Design". Applied Research Laboratory, Penn State University. 26 (05), (1996)

Broderick, C. L., Instructional Systems Design: What it's all about. Training Journal-ELY-, 25-27. (2001)

Bybee R.W. Scientific Inquiry and Science Teaching. En: Flick, L. y Lederman N. (eds.), Scientific inquiry and nature of science: Implications for teaching, learning, and teacher education, Holanda, Kluwer Academic Publishers, pp. 1-14, (2004)

Coll, C., Onrubia, J. y Mauri, T., Tecnología y prácticas pedagógicas: las TIC como instrumentos de mediación de la actividad conjunta de profesores y estudiantes. Anuario de Psicología, 38(3), 377-400. (2007)

Dias, S. B., Diniz, J. A., y Hadjileontiadis, L. J., Embracing and embedding techno-pedagogical strategies. https://doi.org/10.1007/978-3-319-02078-5 3, In Towards an Intelligent Learning Management System Under Blended Learning Springer, Cham. pp. 35-51, (2014)

Freire, P. y Faundez, A., Por una pedagogía de la pregunta: crítica a una educación basada en respuestas a preguntas inexistentes. Buenos Aires: Siglo Veintiuno Editores. (2013)

Furman, M.G., Educar mentes curiosas: la formación del pensamiento científico y tecnológico en la infancia: documento básico, XI Foro Latinoamericano de Educación. (2016)

García S.M. y Furman M.G., Categorización de preguntas formuladas antes y después de la enseñanza por indagación. Praxis \& saber, 5(10), 75-91. (2014)

González, Y.Y. y Díaz, J.G. Integración de la metodología basada en la indagación para mediar la enseñanza de la asignatura Fundamentos de Administración soportada con la herramienta Smile. Boletín Redipe, 4(12), 101-112. (2015)

Johnson, D.W., Johnson R.T. y Stanne M.B. Cooperative Learn-ing methods: A MetaAnálysis. Cooperative Learning Center at the University of Minnesota. (2000)

Kim, P.H., Action research approach on mobile learning design for the underserved. http://doi.org/10.1007/s11423-0089109-2, Educational Technology Research y Development, 57, 415-435. (2009)

Kim, P.H., y An, J. Y. New evaluation vector through the Stanford Mobile Inquiry-Based Learning Environment (SMILE) for participatory action research. Healthcare Informatics Research, 22(3), 164-171. (2016)

Lim, K. Y., Song, B. H., y Kho, M.X., Exploring the Change in Nature and Efficacy of Learners' Questions Through Progressive Interaction with the Stanford Mobile Inquiry-based Learning Environment (SMILE). In Innovations in Educational Change (pp. 225-251). Springer, Singapore. (2019). (2019)

Lee, V.S., What is inquiry-guided learning?. doi:10.1002/tl.20002, New directions for teaching and learning, 129, 5-14 (2012)

Mesa, J.M., Álvarez, J. V., Villanueva, J. M, y de Cos, F. J. Actualización de Métodos de Enseñanza-Aprendizaje en Asignaturas de Dirección de Proyectos de Ingeniería. https://dx.doi.org/10.4067/S0718-50062008000400004, Formación universitaria, 1(4), 23-28.. (2008)

Oliveira, A.W., "Kindergarten, can I have your eyes and ears?" politeness and teacher directive choices in Inquiry-based science classrooms, Cultural studies of Science Education, 4, 803-846, (2009)

Pineda Corcho, A. F. Modelo tecno-pedagógico basado en ludificación y programación competitiva para el diseño de cursos de programación. Ingeniería de Sistemas e Informática. (2014)

Raes, A., Schellens, T., De Wever, B. y Vanderhoven, E., Scaffolding information problem solving in web-based collaborative inquiry learning, Computers \& Education, 59(1), 82-94 (2012)

Reyes-Cárdenas F. y Padilla K., La indagación y la enseñanza de las ciencias. Educación química, 23(4), 415-421. (2012)

Richey, R. C., Fields, D. C., y Foxon, M. Instructional design competencies: The standards. Syracuse, NY: Syracuse University, ERIC Clearinghouse on Information \& Technology. (2001)

Sadker, M., y Sadker, D. Técnicas para la elaboración de preguntas. Estrategias de la enseñanza, 173-228. (1998)

Schwartz, R.S., Lederman, N.G. y Crawford B.A., Developing views of nature of science in an authentic context: An explicit approach to bridging the gap between nature of science and scientific inquiry, Science Education, 610-645, (2004)

Suárez, Á. y otros cuatros autores, Review of the types of mobile activities in mobile inquiry-based learning, Computers \& Education , 118, 38-55 (2018)

Seol, S., Sharp, A., y Kim, P.H., Stanford Mobile Inquiry-based Learning Environment (SMILE): using mobile phones to promote student inquires in the elementary classroom. In Proceedings of the International Conference on Frontiers in Education: Computer Science and Computer Engineering (FECS) (p. 1). The Steering Committee of The World Congress in Computer Science, Computer Engineering and Applied Computing (WorldComp). (2011)

Song, D., Kim, P., y Karimi, A., Inquiry-based learning environment using mobile devices in math classroom. Proceedings of the Association for Education Communications and Technology, 2012. Kentucky, 386-392. (2012)

Uno, G.E., Inquiry in the classroom, https://doi.org/10.2307/1311488, BioScience, 40(11), 841- 843, (1990)

Yeh, Y. F., Hsu, Y. S., Wu, H. K., y Chien, S. P. Exploring the structure of TPACK with video-embedded and disciplinefocused assessments. https://doi.org/10.1016/i.compedu.2016.10.006, Computers and Education, 104, 49-64. (2017) 